Bull. Austral. Math. Soc.

$58 \mathrm{~F} 10,54 \mathrm{H} 20$

VOL. 69 (2004) [63-68]

\title{
POINTWISE CHAIN RECURRENT MAPS OF THE TREE
}

\author{
Zhang Gengrong and Zeng Fanping
}

Let $T$ be a tree, $f: T \rightarrow T$ be a continuous map. We show that if $f$ is pointwise chain recurrent (that is, every point of $T$ is chain recurrent under $f$ ), then either $f^{a_{n}}$ is identity or $f^{a_{n}}$ is turbulent if $\operatorname{Fix}(f) \cap \operatorname{End}(T)=\emptyset$; or else $f^{a_{n-1}}$ is identity or $f^{a_{n-1}}$ is turbulent if $\operatorname{Fix}(f) \cap \operatorname{End}(T) \neq \emptyset$. Here $n$ denotes the number of endpoints of $T$ and, $a_{n}$ denotes the minimal common multiple of $2,3, \ldots, n$.

\section{INTRODUCTION}

Firstly some notation and definitions are established. Let $(X, d)$ be a compact metric space and $g: X \rightarrow X$ be a continuous map. If $g^{n}(x)=x \neq g^{k}(x), k=1,2, \ldots, n-1$, for some $x \in X$ and some positive integer $n$, then the point $x$ is called a periodic point of period $n$, where $g^{0}=i d, g^{i}=g \circ g^{i-1}(i \geqslant 1)$. In particular, if $g(x)=x$, then $x$ is called a fixed point of $g$, the set of all fixed points of $g$ is denoted by $\operatorname{Fix}(g)$. For $x, y \in X$ and $\varepsilon>0$, an $\varepsilon$-chain from $x$ to $y$ is a finite sequence $x=x_{0}, x_{1}, \ldots, x_{n-1}, x_{n}=y$ with $d\left(g\left(x_{i}\right), x_{i+1}\right)<\varepsilon$ for $0 \leqslant i \leqslant n-1$. We say $x$ chains to $y$ under $g$, if for each $\varepsilon>0$, there is an $\varepsilon$-chain from $x$ to $y$. A point $x$ is said to be chain recurrent if $x$ chains to itself. The map $g$ is said to be pointwise chain recurrent if every point of $X$ is chain recurrent under $g$. The following facts about chain recurrent are standard observations:

(a) If $g$ is pointwise chain recurrent, then $g$ maps $X$ onto $X$.

(b) $g$ is pointwise chain recurrent if and only if $g^{n}$ is pointwise chain recurrent for every $n>0$.

(c) [3, Theorem A] If $X$ is connected and $g: X \rightarrow X$ is pointwise chain recurrent, then there is no nonempty open set $U \neq X$ such that $g(\bar{U}) \subset U$.

Being chain recurrent is an important dynamical property of a system and has been studied intensively in recent years. For more details see $[1,2,3,4,5,7,8]$.

A tree is any space which is uniquely arcwise connected and homeomorphic to the union of finitely many copies of the unit interval, that is, a graph(see [6])containing no

Received 2nd June, 2003

Project supported by NSFCs $(10361001,10226014)$ and supported partly by GuangXi Science Foundation (0229001, 0249002).

Copyright Clearance Centre, Inc. Serial-fee code: 0004-9727/04 \$A2.00+0.00. 
cycles. Let $T$ be a tree, the points of $T$ which have no neighbourhood homeomorphic to an open interval of the real line are called vertices, written by $V(T)$. Let $z \in T$, the number of connected components of $T \backslash\{z\}$ is called the valence of $z$. A vertex of valence 1 is called an endpoint of $T$ and a vertex of valence larger than 2 is called a branching point of $T$. Denote by $\operatorname{End}(T)$ and $\operatorname{Br}(T)$ the sets of endpoints and branching points of $T$, respectively, and let $\mathrm{NE}(T)$ be the number of endpoints of $T$. For a point $p \in \operatorname{Fix}(f)$, the connected component of $\operatorname{Fix}(f)$ which contains $p$ is represented by $C_{p}$. Let $N$ be the set of positive integers. For any $n \in N$ with $n \geqslant 2$, let $a_{n}$ denote the minimal common multiple of $2,3, \ldots, n$.

For $a, b \in T$, we use $[a, b]$, to denote the smallest closed connected subset containing $a$ and $b$. We define $(a, b]=[a, b] \backslash\{a\}$ and similarly define $(a, b)$ and $[a, b)$. We also use $T_{b}(a)$ to denote the connected component of $T \backslash\{b\}$ which contains $a$. For a subset $A$ of $T$, we use $\operatorname{int}(A), \bar{A}$ and $\partial(A)$ to denote the interior, the closure and the boundary of $A$, respectively.

A map $g: T \rightarrow T$ is called turbulent if there are closed non-degenerate connected subsets $J$ and $K$ with disjoint interiors such that $g(J) \cap g(K) \supset J \cup K$.

The following are obviously:

(1) If $g$ is turbulent then $g^{n}$ is turbulent for any $n>1$.

(2) If there exist $p \in \operatorname{Fix}(g), y \in T$ such that $y \in\left((g(y), p)\right.$ and $p=g^{2}(y)$, then $g$ is turbulent.

In [2], it is proved that a pointwise chain recurrent map $h$ of the interval must satisfy that either $h^{2}$ is the identity or $h^{2}$ is turbulent. In [4], it is shown that a pointwise chain recurrent map $h$ of the space $Y$ satisfy that either $h^{12}$ is identity or $h^{12}$ is turbulent.

In this paper, we prove the following:

MAIN TheOREM. Let $T$ be a tree with $n$ endpoints, $f: T \rightarrow T$ be a continuous map. If $f$ is pointwise chain recurrent, then

(1) If $\operatorname{Fix}(f) \cap \operatorname{End}(T)=\emptyset$, then either $f^{a_{n}}$ is the identity or $f^{a_{n}}$ is turbulent;

(2) If $\operatorname{Fix}(f) \cap \operatorname{End}(T) \neq \emptyset$, then either $f^{a_{n-1}}$ is the identity or $f^{a_{n-1}}$ is turbulent.

From the Main Theorem, we obtain the following Corollary, which sharpens the result of [4].

COROLlary. Let $f$ be a pointwise chain recurrent map of the space $Y$, then

(1) If $\operatorname{Fix}(f) \cap \operatorname{End}(T) \neq \emptyset$, then either $f^{2}$ is turbulent or $f^{2}$ is identity;

(2) If $\operatorname{Fix}(f) \cap \operatorname{End}(T)=\emptyset$, then either $f^{6}$ is identity or $f^{6}$ is turbulent.

\section{Proof of MaIN Theorem}

Before proving the main theorem, some lemmas are established. 
Lemma 2.1. Suppose $k, m, n \in N$ with $k m \leqslant n$, then $k a_{m} \leqslant a_{n}$ and $k a_{m} \mid a_{n}$.

Proof: Let $a_{m}=\prod_{i=1}^{s+l} q_{i}^{r_{i}}$ and $k=\prod_{i=1}^{l} q_{i}^{t_{i}}$, where $q_{i}$ are prime number with $q_{i} \neq q_{j}$, for all $1 \leqslant i<j \leqslant s+l$. Then $k a_{m}=\prod_{i=1}^{l} q_{i}^{r_{i}+t_{i}} \prod_{j=l+1}^{s+l} q_{j}^{r_{j}}$. Obviously, $q_{i}^{r_{i}+t_{i}} \leqslant n$ for all $i<l+1$, and $q_{i}^{r_{i}} \leqslant m$ for all $l<i<l+s+1$. Thus, $k a_{m} \mid a_{n}$, which completes the proof.

Lemma 2.2. Let $T$ be a tree, $f: T \rightarrow T$ be a pointwise chain recurrent map. Then $\operatorname{Fix}(f) \cap \operatorname{int}(T) \neq \emptyset$. In particular, if $\operatorname{Fix}(f)=\{p\}$, then $p \notin \operatorname{End}(T)$.

Proof Suppose that $\operatorname{Fix}(f) \cap \operatorname{int}(T)=\emptyset$, that is $\operatorname{Fix}(f) \subset \operatorname{End}(T)$. Given $v_{1}$ $\in \operatorname{int}(T)$, then $f\left(v_{1}\right) \in\left(v_{1}, e_{1}\right)$ for some endpoint $e_{1}$.

(1) If $\left(v_{1}, e_{1}\right) \cap \operatorname{Br}(T)=\emptyset$, then $\left(v_{1}, e_{1}\right] \cap \operatorname{Fix}(f)=\left\{e_{1}\right\}$ and $f\left(\left[v_{1}, e_{1}\right]\right) \subset\left(v_{1}, e_{1}\right]$. But this contradicts (c). Hence there exists some point $v_{2} \in\left(v_{1}, e_{1}\right) \cap \operatorname{Br}(T)$ satisfying $\left(v_{1}, v_{2}\right) \cap \operatorname{Br}(T)=\emptyset$.

(2) Obviously $v_{2} \in\left(v_{1}, f\left(v_{2}\right)\right)$. Then there exists some endpoint $e_{2}$ such that $f\left(v_{2}\right)$ $\in\left(v_{2}, e_{2}\right)$. Hence there exists some point $v_{3} \in\left(v_{2}, e_{2}\right) \cap \operatorname{Br}(T)$ satisfying $\left(v_{2}, v_{3}\right) \cap \operatorname{Br}(T)$ $=\emptyset$.

(3) Thus, we can find infinite points $v_{1}, v_{2}, v_{3}, \ldots$ satisfy: $v_{i+1} \in \operatorname{Br}(T),\left(v_{i}, v_{i+1}\right)$ $\cap \operatorname{Br}(T)=\emptyset, v_{i} \neq v_{j}$ for all $i, j \in N$ and $i \neq j$. This is contrary to the fact that $\operatorname{Br}(T)$ is finite, which completes the proof.

LEMMA 2.3. Let $T$ be a tree, $f: T \rightarrow T$ be a pointwise chain recurrent map. $p \in \operatorname{End}(T) \cap \operatorname{Fix}(f)$. If $C_{p} \cap \operatorname{Br}(T)=\emptyset$, then either $f^{-1}\left(C_{p}\right) \cap T \backslash \operatorname{Fix}(f) \neq \emptyset$ or $f$ is turbulent.

Proof: Without loss of generality, let $C_{p}=\{p\}$. Suppose $f^{-1}(p)=\{p\}$.

CASE 1. There is some point $c$ with $(p, c) \cap(\operatorname{Fix}(f) \cup \operatorname{Br}(T))=\emptyset$. If $f(x) \in(p, x)$ for all $x \in(p, c)$, then $f([p, x]) \subset[p, x)$ for all $x \in(p, c)$. That is a contradiction. Thus, $x \in(p, f(x))$ for all $x \in(p, c)$, and there exists some point $b \in(p, c)$ with $f(x) \in T_{b}(c)$ for all $x \notin T_{c}(b)$. Thus $f\left(\overline{T_{b}(c)}\right) \subset T_{b}(c)$, also a contradiction.

CASE 2. There are some fixed points $p_{0}, p_{1}, \ldots$ with $\left(p_{0}, p\right) \cap \operatorname{Br}(T)=\emptyset, d\left(p_{i}, p\right)$ $<d\left(p_{i-1}, p\right)$ for all $i \in N$ and $\lim _{i \rightarrow \infty} d\left(p_{i}, p\right)=0$. Without loss of generality, we assume $\left(p_{i}, p_{i+1}\right) \cap \operatorname{Fix}(f)=\emptyset$. If there exists some positive integer $i_{0}$ such that $f(x) \in[p, x]$ for all $x \in\left[p, p_{i_{0}}\right]$ or $x \in[p, f(x)]$ for all $x \in\left[p, p_{i_{0}}\right]$, we obtain a analogous contradiction as case 1. Then, there exists some $i_{0} \in N$ satisfies $f(x) \in(p, x)$ for all $x \in\left(p_{i_{0}+1}, p_{i_{0}}\right)$ and $x \in(p, f(x))$ for all $x \in\left(p_{i_{0}+1}, p_{i_{0}+2}\right)$. If $f(x) \neq p_{i_{0}+2}$ for all $x \in\left(p_{i_{0}+1}, p_{i_{0}}\right)$ and $f(x) \neq p_{i_{0}}$ for all $x \in\left(p_{i_{0}+1}, p_{i_{0}+2}\right)$, then there some open set $U \subset\left(p_{i_{0}}, p_{i_{0}+2}\right)$ such that $f(\bar{U}) \subset U$. That is a contradiction. Without loss of generality, we assume $y \in\left[p_{i_{0}}, p_{i_{0}+1}\right]$ with $f(y)=p_{i_{0}+2}$ and $f^{-1}\left(p_{i_{0+2}}\right) \cap\left(p_{i_{0}+1}, y\right)=\emptyset$. If $f(x) \in\left(p_{i_{0}+2}, b\right)$ for all $x \in\left(p_{i_{0}+2}, p_{i_{0}+1}\right)$ and some $b \in\left(p_{i_{0+1}}, y\right)$, then $f(\bar{U}) \subset U$ for some open set $U \subset\left(p_{i_{0}+2}, b\right)$. that is a contradiction. Else, $f(x)=y$ for some $x \in\left(p_{i_{0}+2}, p_{i_{0}+1}\right)$, then $f$ is turbulent. This completes 
the proof.

LEMma 2.4. Let $[p, c]$ be an interval, $g \in C^{0}[p, c]$ be a pointwise chain recurrent map. If $\{p, c\} \cap \operatorname{Fix}(g) \neq \emptyset$, then $g$ is turbulent or $g$ is identity.

Proof: Suppose that $g$ is not turbulent and $g$ is not identity and $p \in \operatorname{Fix}(g)$. Without loss of generality, we assume that $C_{p}=\{p\}$. Then, by Lemma $2.3, f(y)=p$ for some $y \in(p, c]$. There exists some point $b \in(p, y)$ with $f(x) \in[p, b)$ for all $x \in[p, y]$. Hence $f([p, y]) \subset[p, y)$, a contradiction, which completes the proof.

LEMMA 2.5. Let $T$ be a tree, $f: T \rightarrow T$ be a pointwise chain recurrent map. If $n>2$ and $f^{-1}(\operatorname{Fix}(f)) \cap(T \backslash \operatorname{Fix}(f))=\emptyset$, then either $\operatorname{Card}(\operatorname{End}(\bar{H}))<n$ for each connected component $H$ of $T \backslash \operatorname{Fix}(f)$ or $f^{2}$ is turbulent.

Proof Suppose that there exists some connected component $H$ of $T \backslash$ Fix $(f)$ satisfied that $\bar{H}$ has $n$ endpoints and let $T \backslash H=H_{1}$.

CASE 1. If $\operatorname{Card}(\operatorname{Fix}(f)) \geqslant 2$, then $\operatorname{Card}(\bar{H} \cap \operatorname{Fix}(f)) \geqslant 2$ or $\operatorname{Card}\left(H_{1} \cap \operatorname{Fix}(f)\right) \geqslant 2$.

SubCase 1.1. Card $(\bar{H} \cap \operatorname{Fix}(f)) \geqslant 2$, then $f(\bar{H})=\bar{H}$. But $\operatorname{Fix}(f) \cap \bar{H} \subset \operatorname{End}(\bar{H})$, this contradicts Lemma 2.2 .

SubCASE 1.2. Card $\left(H_{1} \cap \operatorname{Fix}(f)\right) \geqslant 2$ and Card $(H \cap \operatorname{Fix}(f))=1$, then $H_{1}$ is connected set and $\operatorname{int}\left(H_{1}\right) \cap \operatorname{Fix}(f) \neq \emptyset$. Then $f\left(H_{1}\right)=H_{1}$, hence $f(\bar{H})=\bar{H}$. Also a contradiction.

CASE 2. If $\bar{H} \cap H_{1}=\operatorname{Fix}(f)=\{p\}$, then $f(\bar{H})=\bar{H}, f\left(H_{1}\right)=H_{1}$ or $f(\bar{H})=H_{1}$, $f\left(H_{1}\right)=\bar{H}$.

SUBCASE 2.1. If $f(\bar{H})=\vec{H}$ and $f\left(H_{1}\right)=H_{1}$, we have a contradiction as above.

SUBCASE 2.2. if $f(\bar{H})=H_{1}$ and $f\left(H_{1}\right)=\bar{H}$, then we have $f^{2}\left(H_{1}\right)=H_{1}$. Let $g=\left.f^{2}\right|_{H_{1}}$, it is not difficult to see that $g$ is a not identity. Then, by Lemma $2.4, g$ is turbulent and $f^{2}$ is turbulent. This completes the proof.

LEMMA 2.6. Let $T$ be a tree, $f: T \rightarrow T$ be a pointwise chain recurrent map and, $f$ is not identity. If $f^{-1}(\operatorname{Fix}(f)) \cap(T \backslash \operatorname{Fix}(f))=\emptyset$, then

(1) $\operatorname{Fix}(f)$ is a connected set and;

(2) If $\operatorname{Card}(\operatorname{Fix}(f))>1$, then $\partial(\operatorname{Fix}(f)) \subset \operatorname{Br}(T) \cup \operatorname{End}(T)$ and;

(3) If $\operatorname{Fix}(f) \cap \operatorname{End}(T) \neq \emptyset$, then $\operatorname{Fix}(f) \cap \operatorname{Br}(T) \neq \emptyset$.

Proof: (1) If Fix $(f)$ is not a connected set, then, there is a connected component $H$ of $T \backslash \operatorname{Fix}(f)$ such that $\operatorname{Card}(\partial(H) \cap \operatorname{Fix}(f))>1$. We have $f(\bar{H})=\bar{H}$ and $\operatorname{Fix}(f) \cap H$ $=\emptyset$. This is a contradiction, by Lemma 2.2 .

(2) Suppose $\operatorname{Card}(\operatorname{Fix}(f))>1$. If there exists some point $p \in \partial(\operatorname{Fix}(f)) \backslash(\operatorname{Br}(T)$ $\cup \operatorname{End}(T)$ ), let $H$ be the unique connected component of $T \backslash \operatorname{Fix}(f)$ such that $p \in \bar{H}$. Then $f(\bar{H})=\bar{H}$ and $\operatorname{Fix}(f) \cap \bar{H} \subset \operatorname{End}(\bar{H})$. This is a contradiction, by Lemma 2.2.

(3) If $p \in \operatorname{Fix}(f) \cap \operatorname{End}(T)$ and $\operatorname{Fix}(f) \cap \operatorname{Br}(T)=\emptyset$, then, by (2), $\operatorname{Fix}(f)=\{p\}$. But this contradicts Lemma 2.2. This completes the proof. 
ProOF of MAIN TheOREM: We do argument on induction.

If $n=2$, by [3] and Lemma 2.4, we know that the assertion is true.

Inductively, we assume that the assertion is true for all $T$ with endpoints less than $n$ and $n>2$. Now suppose $f$ is not the identity and continue argument for $T$ with $n$ endpoints in the following two cases.

CASE 1. $f^{-1}(\operatorname{Fix}(f)) \cap(T \backslash \operatorname{Fix}(f)) \neq \emptyset$. In this case the following two subcases are considered.

SUBCASE 1.1. Fix $(f) \cap \operatorname{End}(T)=\emptyset$ Without loss of generality, let $f\left(z_{1}\right)=p \in \operatorname{Fix}(f)$ and $z_{1} \neq p$. Denotes $C_{0}=\left\{z_{1}\right\}$.

(1) $f^{-1}\left(z_{1}\right) \cap T_{z_{1}}(p) \neq \emptyset$. Otherwise, there is some nonempty open set $U \subset T_{z_{1}}(p)$ with $f(\bar{U}) \subset U$. A contradiction.

(2) Let $B_{1}=\left\{z_{1}^{1}, z_{1}^{2}, \ldots, z_{1}^{k_{1}}\right\} \subset f^{-1}\left(z_{1}\right) \cap T_{z_{1}}(p)$ with $\left(z_{1}^{i}, p\right) \cap f^{-1}\left(z_{1}\right)=\emptyset$, for all $1 \leqslant i \leqslant k_{1}$ and $B_{1}$ is the largest set with this property. Let $A_{1}$ denote $T_{z_{1}}(p) \cap T_{z_{1}^{1}}(p)$ $\cap T_{z_{1}^{2}}(p) \cap \ldots \cap T_{z_{1}^{k_{1}}}(p)$. Then $f^{-1}\left(B_{1}\right) \cap A_{1} \neq \emptyset$ (Else, there is some connected open subset $U$ with $f(\bar{U}) \subset U$, which is a contradiction). Denote $f\left(A_{1}\right) \cap B_{1}=C_{1}$.

(3) Let $B_{2}=\left\{z_{2}^{1}, z_{2}^{2}, \ldots, z_{2}^{k_{2}}\right\} \subset f^{-1}\left(B_{1}\right) \cap A_{1}$ with $\left(z_{2}^{i}, p\right) \cap f^{-1}\left(B_{1}\right)=\emptyset$, for all $1 \leqslant i \leqslant k_{2}$ and $B_{2}$ is the largest set with this property. Let $A_{2}$ denote $T_{z_{2}^{1}}(p) \cap T_{z_{2}^{2}}(p)$ $\cap \ldots \cap T_{z_{1}}(p) \cap A_{1}$. Then $f^{-1}\left(B_{2}\right) \cap A_{2} \neq \emptyset$. Denote $f\left(A_{2}\right) \cap B_{2}=C_{2}$.

(4) By a repetition of this process we can get nonempty sets $\left\{A_{i}, B_{i}, C_{i}\right\}_{i=1}^{\infty}$ with $f\left(A_{i}\right) \cap B_{i}=C_{i}$ and $C_{i+1} \subset B_{i+1} \subset A_{i} \cap f^{-1}\left(B_{i}\right) \subset f^{-1}\left(C_{i}\right)$ for all $i \geqslant 0$. Take some point $z_{n+1} \in C_{n}$, then $z_{i}=f^{n-i+1}\left(z_{n+1}\right) \in C_{i-1}$ for all $1 \leqslant i \leqslant n+1$. Obviously, $z_{j} \in T_{z_{i}}(p)$ for all $i<j$ and $T=\bigcup_{i=1}^{n}\left[p, e_{i}\right]$, where $e_{1}, e_{2}, \ldots e_{n} \in \operatorname{End}(T)$. Thus, $z_{i_{0}}, z_{j_{0}} \in\left[p, e_{i}\right]$ for some $1 \leqslant j_{0}<i_{0} \leqslant n+1$ and $i \leqslant n$. Then $z_{i_{0}} \in\left(p, z_{j_{0}}\right)$.

Denote $k$ the minimal common multiple of $i_{0}-j_{0}$ and $j_{0}$, and Let $g=f^{k}$. There is a point $w \in\left(p, z_{j_{0}}\right)$ with $g(w)=z_{j_{0}}$, since $z_{i_{0}} \in\left(z_{j_{0}}, p\right)$ and $f^{i_{0}-j_{0}}\left(z_{i_{0}}\right)=z_{j_{0}}$. Then $w \in\left(p, z_{j_{0}}\right), g(w)=z_{j_{0}}$ and $g\left(z_{j_{0}}\right)=p \in \operatorname{Fix}(g)$. It follows that $g$ is turbulent. Thus $f^{a_{n}}$ is turbulent, since $k \mid a_{n}$.

SubCase 1.2. If $\operatorname{Fix}(f) \cap \operatorname{End}(T) \neq \emptyset$, let $p \in \operatorname{Fix}(f) \cap \operatorname{End}(T)$.

If $f\left(z_{1}\right)=z_{0}$ for some $z_{0} \in C_{p}$ and $z_{1} \notin C_{p}$, then, taken the process as subcase 1.1, we shall get points $z_{0}, z_{1}, \ldots, z_{n}, \ldots$ with $f\left(z_{i+1}\right)=z_{i}$ for all $i \geqslant 0, z_{j} \in T_{z_{i}}\left(z_{0}\right)$ for all $1 \leqslant i<j$. Obviously $T=\bigcup_{i=1}^{n}\left[z_{0}, e_{i}\right]$, where $e_{1}, e_{2}, \ldots e_{n} \in \operatorname{End}(T)$ and $e_{n}=p$. Then, $z_{i_{0}}, z_{j_{0}} \in\left[z_{0}, e_{i}\right]$ for some $1 \leqslant j_{0}<i_{0} \leqslant n$ and $i \leqslant n-1$, since $\left[z_{0}, p\right] \subset \operatorname{Fix}(f)$. Then $z_{i_{0}} \in\left(z_{j_{0}}, z_{0}\right), i_{0}-j_{0} \leqslant n-1$ and $1 \leqslant j_{0}<n-1$. Let $k$ be the minimal common multiple of $i_{0}-j_{0}$ and $j_{0}$, and Let $g=f^{k}$. There is a point $w \in\left(p, z_{j_{0}}\right)$ with $g(w)=z_{j_{0}}$, since $z_{i_{0}} \in\left(z_{j_{0}}, z_{0}\right)$ and $f^{i_{0}-j_{0}}\left(z_{i_{0}}\right)=z_{j_{0}}$. Then $w \in\left(p, z_{j_{0}}\right), g(w)=z_{j_{0}}$ and $g\left(z_{j_{0}}\right)=z_{0} \in \operatorname{Fix}(g)$. It follows that $g$ is turbulent. Thus $f^{a_{n-1}}$ is turbulent, since $k \mid a_{n-1}$.

If $f^{-1}\left(z_{0}\right)=\left\{z_{0}\right\}$ for all $z_{0} \in C_{p}$, then, there exists some fixed point $q \notin C_{p}$ with 
$f^{-1}(q) \cap T \backslash \operatorname{Fix}(f) \neq \emptyset$. Let $H$ be the connected component of $T \backslash C_{p}$ which contains point $q$, then, $f(\bar{H})=\bar{H}$. Let $\{w\}=C_{p} \cap \bar{H}$. It is obviously that $\{w\} \cap \operatorname{Br}(\bar{H})=\emptyset$ and $f^{-1}(w) \cap \bar{H}=\{w\}$, then $f$ is turbulent, by Lemma 2.3. It follows that $f^{a_{n-1}}$ is turbulent. CASE 2. $f^{-1}(\operatorname{Fix}(f)) \cap(T \backslash \operatorname{Fix}(f))=\emptyset$.

Let $G_{1}, G_{2}$ be two connected components of $T \backslash \operatorname{Fix}(f)$. Then $f\left(\overline{G_{1}}\right)=\overline{G_{2}}$ and $\overline{G_{1}} \cap \overline{G_{2}} \subset \operatorname{Fix}(f)$, if $f\left(G_{1}\right) \cap G_{2} \neq \emptyset$.

If $f^{2}$ is turbulent, then $f^{a_{n-1}}$ is turbulent.

Now we suppose $f^{2}$ is not turbulent, then, by Lemma 2.5 and Lemma 2.6, $\mathrm{NE}(\bar{H})<n$ and $\operatorname{Card}(\bar{H} \cap \operatorname{Fix}(f))=1, f(\bar{H}) \cap \bar{H} \subset \operatorname{Fix}(f)$ for each connected component $H$ of $T \backslash \operatorname{Fix}(f)$.

Given $G$ a connected component of $T \backslash \operatorname{Fix}(f)$. Without loss of generality, we assume that (1) $f^{k}(\bar{G})=\bar{G}$ and $k$ be the minimal positive integer with this property; (2) $m=\operatorname{NE}(\bar{G}) \leqslant \operatorname{NE}\left(f^{j}(\bar{G})\right)$ for all $k \geqslant j \geqslant 1$. Obviously $m<n$. Then either $\left(\left.f^{k}\right|_{\bar{G}}\right)^{a_{m-1}}$ is identity or $\left(\left.f^{k}\right|_{\bar{G}}\right)^{a_{m-1}}=\left.f^{k a_{m-1}}\right|_{\bar{G}}$ is turbulent, by induction. And then either $\left(\left.f^{k}\right|_{f^{j}(\bar{G})}\right)^{a_{m-1}}$ is identity or $\left.f^{k a_{m-1}}\right|_{f^{j}(\bar{G})}$ is turbulent for all $k \geqslant j \geqslant 0$. Since $\bar{G}$ is arbitrary and $k(m-1) \leqslant n$, it follows that either $f^{a_{n}}$ is identity or $f^{a_{n}}$ is turbulent, by Lemma 2.1 .

In particular, if $\operatorname{Fix}(f) \cap \operatorname{End}(T) \neq \emptyset$, then, by Lemma $2.6, k(m-1) \leqslant n-1$. Thus, we have either $f^{a_{n-1}}$ is identity or $f^{a_{n-1}}$ is turbulent, by Lemma 2.1. This completes the proof.

\section{REFERENCES}

[1] L. Block and W.A. Coppel, Dynamics in One Dimension, Lecture Notes in Math. 1513 (Spinger-Verlag, Berlin, Heidelberg, New York, 1991).

[2] L. Block and E.M. Coven, 'Maps of the interval with every point chain recurrent', Proc. Amer. Math. Soc. 98 (1986), 513-515.

[3] L. Block and J. Franke, 'The chain recurrent set, attractors, and explosions', Ergodic Theory Dynamical System 5 (1985), 321-327.

[4] W.J. Guo, F.P. Zeng and Q.Y. Hu, 'Pointwise chain recurrent maps of the space Y', Bull. Austral. Math. Soc. 67 (2003), 79-85.

[5] T. Li and X.D. Ye, 'Chain recurrent point of a tree map', Bull. Austral. Math. Soc. 59 (1999), 181-186.

[6] S.B. Nadler, Continuum theory, an introduction (Marcel Dekker. Inc., New York, 1992).

[7] X.D. Ye, 'The centre and the depth of the centre of a tree map', Bull. Austral. Math. Soc. 48 (1993), 347-350.

[8] F.P. Zeng, H. Mo, W.J. Guo and Q.J. Gao, 'w-limit set of a tree map', Northeast. Math. J. 17 (2001), 333-339.

Department of Mathematics

Guangxi University,

Nanning, Guangxi 530004

People's Republic of China 